López Martín, Elena. (Alumna)

Morgado Aguirre, Borja. (Profesor)

Universidad de Murcia, Departamento de Bellas Artes, Grupo de Investigación Prácticas artísticas

activas y ciudadanía.

\title{
Residir.net: debates en torno a la definición de las residencias artísticas virtuales.
}

\section{To reside.net: debates on defining virtual art residencies.}

TIPO DE TRABAJO:

Póster.

PALABRAS CLAVE:

Residencias artísticas virtuales, Internet, movilidad, arte contemporáneo.

KEY WORDS:

Virtual art residencies, the Internet, mobility, contemporary art.

RESUMEN.

\begin{abstract}
Al hablar de arte y movilidad, es imprescindible aludir a los programas de residencias artísticas, que en las últimas décadas han cobrado un especial protagonismo en la experiencia y currículum de los artistas. Dichas residencias promocionan la figura de un artista nómada que se nutre de las distintas vivencias ofrecidas por un mundo transfronterizo fruto de una sociedad cada vez más globalizada. La necesidad de experimentar con el amplio abanico de contextos existentes también lleva a los artistas a reflexionar, investigar y producir experiencias artísticas en el ciberespacio, donde conceptos como "residencia", "movilidad", "espacio" o "comunidad" requieren de cierta recodificación. Con el objetivo de acceder a distintos puntos de vista en torno a lo que se entiende por residencia artística virtual, se ha contactado y enviado un cuestionario con fines indagatorios a aquellas personas físicas e instituciones que han desarrollado o actualmente desarrollan este tipo de iniciativas. Así, el presente póster pretende mostrar características básicas de las residencias artísticas virtuales junto con otros aspectos conflictivos a la hora de definirlas.
\end{abstract}

\section{ABSTRACT.}

When we talk about art and mobility, art residencies programs are a mandatory mention. In the last few decades, these programs have taken an unprecedented major role in artists' curriculum. Art residencies promote the idea of the nomad artist that gets inspiration from the daily experiences offered by a cross-border world resulting from a increasingly globalized society. The need to experiment with a wide range of contexts leads the artist to reflect and produce art experiences in the cyberspace where some concepts such as "to reside", "mobility", "space" or "community" demand a new re-coding. With the aim of meeting different points of view about what a virtual art residency is, some individuals and organizations that have run this kind of programs have been contacted and interviewed. The current poster expects to show some fundamental characteristics about virtual art residencies, as well as some controversial aspects arisen when it comes to define them. 


\section{CONTENIDO.}

\section{INTRODUCCIÓN E IMPORTANCIA TEÓRICA DE ESTA INVESTIGACIÓN:}

Si bien existe abundante información acerca de lo que son las residencias artísticas, numerosos portales divulgativos que albergan miles de convocatorias, así como distintas guías profesionales sobre cómo generar nuevos proyectos de esta índole, cabe destacar que no existe ningún estudio exhaustivo sobre las residencias artísticas virtuales (a partir de ahora RAV). Las únicas referencias profesionales al respecto provienen de Weronica Trojanska con un sucinto texto en el que describe brevemente diez RAV. Sin embargo, su recopilación no incluye la descripción y análisis de otros numerosos programas desarrollados hasta la fecha de publicación de su texto (2013) y dada la veloz e incesante actividad artística en la Red, ha quedado muy desactualizada en estos tres últimos años. Por otro lado, también podemos contar con el proyecto VIRTUAL ARTIST RESIDENCY: A New Model of AiR que consiste en la creación de un blog y una página web donde subir información periódica acerca de este tipo de residencias. No obstante, a excepción de las primeras publicaciones introductorias del proyecto, el resto de la información ofrecida hace referencia a obras de arte en Internet que sorprendentemente no suelen tener ningún vínculo con el desarrollo y/o resultado de ninguna RAV.

Por todo lo anterior, y tras detectar un desconocimiento general sobre este tipo de iniciativas y una necesidad de definir los programas de residencias artísticas virtuales y de reflexionar sobre los mismos, se ha ideado el presente proyecto de investigación.

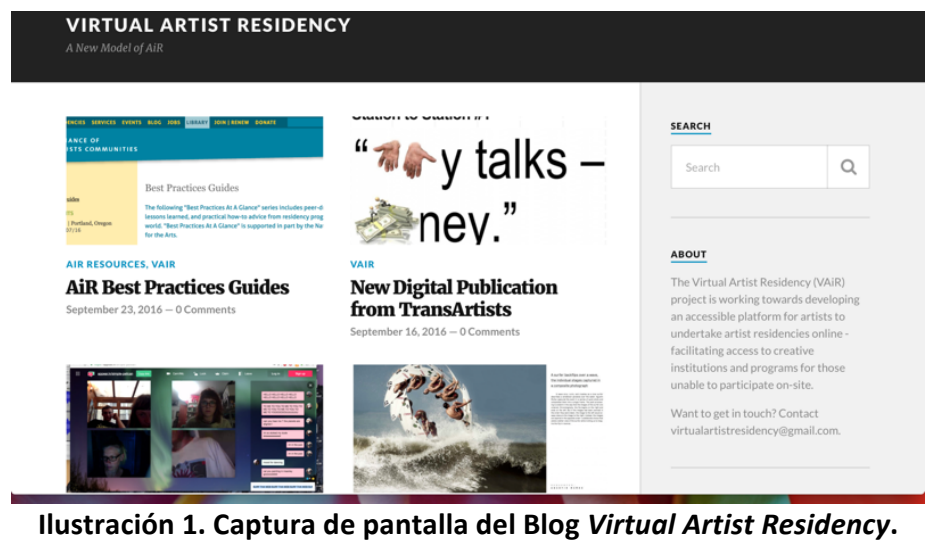

\section{OBJETIVOS:}

- Establecer cuáles son las características básicas de las residencies artísticas virtuales.

- Abrir debates en torno a la definición de estos programas.

- Dar a conocer este tipo de residencias artísticas.

\section{DESARROLLO:}

Al no existir apenas literatura profesional y crítica acerca de los programas de residencias virtuales, se consideró fundamental acudir directamente a los expertos que se habían aventurado a desarrollar tales iniciativas. Por medio de un dilatado y concienzudo trabajo de campo para rastrear todas las RAV que se habían desarrollado hasta el momento (abarcando tanto iniciativas ya concluidas como las más actuales hasta Diciembre de 2016) se localizaron 24 entidades organizadoras en cuyas descripciones oficiales se hacía una clara referencia a las RAV. A la hora de intentar contactarlas surgieron algunas dificultades. Muchas de las iniciativas ya no están vigentes y las organizaciones se han disuelto. Por ello, enlaces y direcciones de contacto se encuentran frecuentemente inactivos. Si bien no podemos saber con certeza si las 24 entidades han recibido nuestra presentación del proyecto, sí se puede afirmar que se han obtenido respuestas de 14 organismos interesados en nuestro proyecto (58,3\% de respuestas) de los cuales 12 ya han remitido unos breves comentarios sobre qué consideran ellos una RAV.

\section{PRINCIPALES CONCLUSIONES Y DEBATES:}

- Se ha generado un recopilatorio de las 24 entidades localizadas que se puede consultar en: ver código QR más abajo (ilustración 2). 


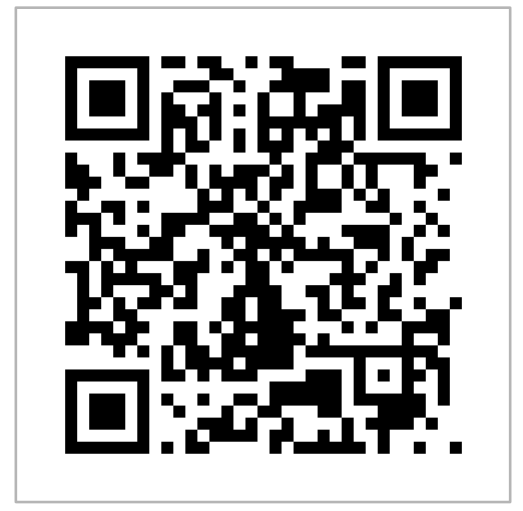

Ilustración 2: Código QR para acceder al repertorio de 24 organizadores de RAV

- Con fines divulgativos, se ha generado un documento online con cada una de las respuestas hasta ahora recibidas que se puede consultar en: ver código QR más abajo (ilustración 3).

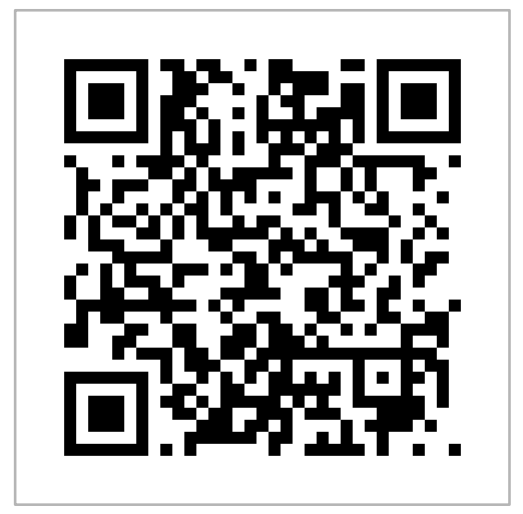
Ilustración 3: Código QR para acceder a los 12 comentarios aclaratorios
acerca de las RAV realizados por los organizadores de las mismas.

\begin{abstract}
- La Red es un lugar en el que la velocidad con la que fluye la información es muy difícil de gestionar por los habitantes del ciberespacio. El entramado del hipertexto se extiende en una red en la que muchos de los nexos se vuelven enlaces rotos. Una misma información puede aparecer duplicada, modificada, contradicha e incluso eliminada en distintos espacios. Esto hace que, a pesar del rigor perseguido por esta investigación durante largos meses de trabajo en los que se han contactado artistas, autores, periodistas y organizaciones, no podamos asegurar que las 24 entidades organizadoras de RAV encontradas sean las únicas existentes, ni que todos los cuestionarios enviados hayan llegado a sus destinatarios. Lo que si podemos asegurar es que se trata de la investigación más completa realizada al respecto hasta ahora.
\end{abstract}

- La mayoría de profesionales coincide en que las RAV funcionan de una manera muy similar a las residencias artísticas ordinarias: un artista ocupa un espacio durante un periodo de tiempo determinado para la realización de un nuevo proyecto artístico.

- En las RAV, los espacios que se "habitan" son virtuales, y al igual que ocurre en el espacio físico, son diversos (web, blog, Facebook, Instagram, Twitter, Tumblr, portales de realidad virtual como Second Life, Minecraft, etc.)

- Al igual que ocurre en las residencias artísticas físicas, el proceso de producción de la obra puede o no involucrar a la comunidad local/virtual. Sin embargo, dado el carácter conectivo de la Red, es más frecuente que las piezas producidas en las RAV requieran de materiales derivados de la actividad de la comunidad virtual en el ciberespacio y/o la participación directa de la misma. Asimismo, existen prácticamente los mismos tipos de residencias físicas que virtuales (centradas en un tema, en una disciplina, $u$ otro objetivo concreto) pero es difícil encontrar en el espacio virtual una residencia que promueva el aislamiento del artista, el retiro a un lugar que quede muy alejado de la rutina como excusa para encontrar cierta inspiración para producir arte. Internet, su ritmo y sus distintos 
espacios y lógicas suelen estar insertados de antemano en el día a día de la vida de los artistas participantes. Lejos de promover aislamiento, las RAV suelen fomentar el encuentro.

- Debates en cuanto a la taxonomía:

- Las 24 iniciativas recogidas aluden en sus bases a las RAV. Sin embargo, entre ellas existen iniciativas que podrían considerarse instalaciones interactivas temporales o exposiciones virtuales (véase Desktop Residency o The Virtual Residency-A call for a virtual migration to the "house Europe").

- Asimismo, existen proyectos denominados RAV (como por ejemplo AND/DaDa artists' online residency programme) de los que, tras consultar con los agentes participantes, se ha sabido que parte de la interacción entre los artistas se ha producido en el espacio físico, al no tratarse de relaciones exclusivamente digitales, de momento, se han dejado fuera de ese repertorio de 24 iniciativas anteriormente mencionado.

- Existen iniciativas en la Red que no se denominan RAV pero que, por el contrario, cumplen todos los requisitos generales de una RAV. ¿Hasta qué punto la taxonomía nos dirige o limita a la hora de clasificar iniciativas virtuales?

\section{FUENTES REFERENCIALES.}

DESKTOP RESIDENCY. S.f. [Consulta: 14/07/2016]. Disponible en: http://www.desktopresidency.com

FURTHERFIELD. AND/DaDa Open Call for a Creative Technologist. 2012. [Consulta: 27/09/2016]. Disponible en:

http://www.furtherfield.org/community/calendar/anddada-open-call-creative-technologist

TROJANSKA, Weronika. "Virtual Art Residencies - a Manual", Interartive. Número especial \#55. 2013. ISSN: 2013-679X [Consulta: 20/08/2016]. Disponible en: http://artmobility.interartive.org/virtual-art-residencies-a-manual-wero

VIRTUAL ART RESIDENCIES: A new Model of AIR. 2015. [Consulta: 22/08/2016]. Disponible en: https://virtualartistresidency.wordpress.com

VIRTUAL RESIDENCY.NET. The Virtual Residency-A call for a virtual migration to the "house Europe". 2006. [Consulta: 03/08/2016]. Disponible en: http://www.virtual-residency.net 
López Martín, Elena; Morgado Aguirre, Borja

Residir.net: debates en torno a la definición de las residencias artísticas virtuales

III CONGRESO INTERNACIONAL DE INVESTIGACIÓN EN ARTES VISUALES :: ANIAV 2017 :: GLOCAL [codificar, mediar, transformar, vivir]

http://dx.doi.org/10.4995/ANIAV.2017.4599

RESIDIR.NET

DEB@TES EN TORNO A LA DEFINICIÓN DE LAS RESIDENCIAS ARTíSTICAS VIRTUALES

Elena López Martín \# Borja Morgado Aguirre - Universidad de Murcia

\section{\# INTRODUCCIÓN}

Evidente escasez de estudios profundos acerca de las residencias artísticas virtuales (RAV). Las únicas breves referencias profesionales al respecto son:

o El breve texto de Weronica Trojanska "Virtual Art Residencies - a Manual" que no incluye la descripción y análisis de otros numerosos programas desarrollados hasta a fecha de publicación de su texto (2013) y que ha quedado muy desactualizada en estos tres últimos años.

- El proyecto VIRTUAL ART RESIDENCIES: A new Model of AIR, web y blog donde subir información periódica acerca de este tipo de residencias. No obstante, a excepción de las primeras publicaciones introductorias del proyecto, el resto de la información ofrecida hace referencia a obras de arte en Internet que sorprendentemente no suelen tener ningún vínculo con el desarrollo y/o resultado de ninguna RAV.

Desconocimiento general de la mayoria de este tipo de iniciativas.

Necesidad de definir los programas de residencias artísticas virtuales y reflexionar sobre los mismos.

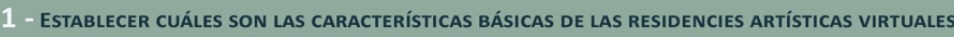

2 - ABRIR UN DEBATE CRítICO EN TORNO A LA DEFINICIÓN DE ESTOS PROGRAMAS.

3 - DAR A CONOCER ESTE TIPO DE RESIDENCIAS ARTÍSTICAS.

\# DESARROLLO

Trabajo de campo para rastrear todas las RAV que se habían desarrollado hasta el momento (abarcando tanto iniciativas ya concluidas como las más actuales hasta Diciembre de 2016).

Localización de 24 entidades organizadoras en cuyas descripciones oficiales se hacía una clara referencia a las RAV.

Puesta en contacto con dichas entidades para preguntárles qué son para ellos las RAV. Problemas derivados: algunas organizaciones e identidades están disueltas.

Obtención de 14 organismos interesados en nuestro proyecto ( $58,3 \%$ de respuestas) de los cuales 12 ya han remitido unos breves comentarios sobre qué consideran ellos una RAV.

\# DEBATES EN CUANTO A LA TAXONOMÍA

- Las 24 iniciativas recogidas aluden en sus bases a las RAV. Sin embargo, algunas podrían considerarse instalaciones temporales o exposiciones virtuales.

- Existen proyectos denominados RAV (ej: AND/DaDa artists' online residency programme) cuyo proceso transcurre en parte en el mundo físico, por lo que se han excluido de nuestro repertorio.

- Existen iniciativas que no se denominan RAV pero que cumplen todos los requisitos generales de una RAV. ¿Hasta qué punto la taxonomía nos dirige o limita a la hora de clasificar iniciativas virtuales?

\section{\# FUENTES}

- DESKTOP RESIDENCY. S.f. [Consulta: 14/07/2016]. Disponible en: http://www.desktopresidency.com

- FURTHERFIELD. AND/DaDa Open Call for a Creative Technologist. 2012. [Consulta: 27/09/2016]. Disponible en: http://www.furtherfield.org/community/calendar/anddada-open-call-creative-technologist

- TROJANSKA, Weronika. "Virtual Art Residencies - a Manual", Interartive. Número especial \#55. 2013. ISSN: 2013-679X [Consulta: 20/08/2016]. Disponible en: http://artmobility.interartive.org/virtual-art-residencies-a-manual-wero

- VIRTUAL ART RESIDENCIES: A new Model of AIR. 2015. [Consulta: 22/08/2016]. Disponible en: https://virtualartistresidencywordpress.com

- VIRTUAL RESIDENCY.NET. The Virtual Residency-A call for a virtual migration to the "house Europe". 2006. [Consulta: 03/08/2016]. Disponible en: http://www.virtual-residency.net

- Creación y publicación de un recopilatorio de las 24 entidades localizadas (QR A) y de un documento online con las definiciones hasta ahora recibidas (QR B). A pesar de no poder asegurar que no existan más iniciativas, se trata de la investigación más completa realizada al respecto.

2 - La mayoría de profesionales coincide en que las RAV funcionan de una manera muy similar a las residencias artísticas ordinarias. Los espacios que se "habitan" son virtuales y diversos.

3 - Es más frecuente que las piezas producidas en las RAV requieran de materiales derivados de la actividad de la comunidad virtual en el ciberespacio y/o la participación directa de la misma.

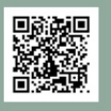

A

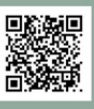

B

\section{\# CONCLUSIONES}

ANIAV Asociación Nacional de Investigación en Artes Visuales

This work is licensed under a Creative Commons Attribution-NonCommercial-NoDerivatives 4.0 International License (CC BY-NC-ND 4.0) 